\title{
Stability of the logistic population model with generalized piecewise constant delays
}

\section{Duygu Aruğaslan* and Leyla Güzel}

\section{*Correspondence:}

duyguarugaslan@sdu.edu.tr Department of Mathematics, Süleyman Demirel University, Isparta, 32260, Turkey

\section{Springer}

\begin{abstract}
In this paper, we consider the logistic equation with piecewise constant argument of generalized type. We analyze the stability of the trivial fixed point and the positive fixed point after reducing the equation into a nonautonomous difference equation. We also discuss the existence of bounded solutions for the reduced nonautonomous difference equation. Then we investigate the stability of the positive fixed point by means of Lyapunov's second method developed for nonautonomous difference equations. We find conditions formulated through the parameters of the model and the argument function. We also present numerical simulations to validate our findings.
\end{abstract}

MSC: 34K20; 34K60; 39A30; 92D25

Keywords: logistic equation; piecewise constant argument; nonautonomous difference equations; boundedness; stability; Lyapunov's second method

\section{Introduction and preliminaries}

Theory of ordinary differential equations plays an important role for solving fundamental problems in population dynamics. However, in some cases, it may not provide consistent results that meet the realities. This fact may arise from the negligence of time delays, impacts or other specific phenomena that are not suitable to be modeled by classical differential equations.

The logistic equation is one of the well-known models that describe the growth of a single species population with limited resources. The general logistic population model is described by

$$
\frac{\mathrm{d} x}{\mathrm{~d} t}=x^{\prime}(t)=(a-b x(t)) x(t)
$$

where $a$ is the intrinsic growth rate and $\frac{a}{b}$ is the carrying capacity of the population, $x(t)$ represents the population density at time $t$. In (1), it is assumed that the growth rate of a population at any time $t$ depends on the number of individuals at that time. In practice, the process of reproduction is not instantaneous. For example, in a Daphnia a large clutch presumably is determined not by the concentration of unconsumed food available when the eggs hatch, but by the amount of food available when the eggs were forming, some time before they pass into the broad pouch. Between this time of determination and the time of

(c) 2015 Aruğaslan and Güzel. This article is distributed under the terms of the Creative Commons Attribution 4.0 International License (http://creativecommons.org/licenses/by/4.0/), which permits unrestricted use, distribution, and reproduction in any medium, provided you give appropriate credit to the original author(s) and the source, provide a link to the Creative Commons license, and indicate if changes were made. 
hatching many newly hatched animals may have been liberated from the brood pouches of other Daphnia in the culture, thus increasing the population [1]. Hutchinson [2] assumed egg formation to occur $\tau$ units of time before hatching and proposed the following more realistic logistic equation:

$$
x^{\prime}(t)=(a-b x(t-\tau)) x(t)
$$

where $a$ and $b$ have the same meaning as in the logistic equation (1), $\tau$ is a positive constant. Equation (2) is known as Hutchinson's equation or (autonomous) delayed logistic equation. There exist many studies about logistic equation with or without time delay [35]. In general, delay differential equations exhibit much more complicated dynamics than ordinary differential equations since a time delay could cause a stable equilibrium to become unstable and lead the populations to fluctuate. There are also several papers [6-8] that study the logistic equation with piecewise constant argument of the following form:

$$
x^{\prime}(t)=(a-b x([t])) x(t)
$$

where [·] denotes the greatest integer function. Here, the distance between the successive switching moments is always constant and equal to 1 . Differential equations with piecewise constant argument $[t]$ was introduced by Cooke and Wiener [9]. Akhmet has generalized the concept of differential equations with piecewise constant argument by taking arbitrary piecewise constant functions as arguments [10] and thus it has been assumed that there is no restriction on the distance between the consecutive switching moments of the argument. Later, this class of differential equations and its applications have been studied by several authors [11-17].

Let $\mathbb{N}$ and $\mathbb{R}^{+}$be the set of natural numbers and nonnegative real numbers, respectively, i.e., $\mathbb{N}=\{0,1,2, \ldots\}, \mathbb{R}^{+}=[0, \infty)$. Denote by $\mathbb{R}^{n}$ the $n$-dimensional real space for a positive integer $n$ and by $\|\cdot\|$ the Euclidean norm in $\mathbb{R}^{n}$. In [6], Altıntan investigated the stability for (3) by reducing it into the following autonomous discrete equation:

$$
x_{k+1}=F\left(x_{k}\right)=x_{k} \mathrm{e}^{a-b x_{k}},
$$

where $x_{k}=x(k), k \in \mathbb{N}$. It is indicated that the trivial fixed point $x=0$ of (4) is asymptotically stable (resp., unstable) if $\mathrm{e}^{a}<1$ (resp., $\mathrm{e}^{a}>1$ ) and the positive fixed point $x=\frac{a}{b}$ of (4) is asymptotically stable (resp., unstable) if $|1-a|<1$ (resp., $|1-a|>1$ ) since $F^{\prime}(0)=\mathrm{e}^{a}$ and $F^{\prime}\left(\frac{a}{b}\right)=1-a$.

Our aim in this paper is to consider the logistic growth model with piecewise constant argument of generalized type given by

$$
x^{\prime}(t)=(a-b x(\beta(t))) x(t),
$$

where $a$ and $b$ are assumed to be nonzero constants of the same sign, $\beta(t)=\theta_{k}$ for $t \in$ $\left[\theta_{k}, \theta_{k+1}\right), k \in \mathbb{N}$ and $\theta_{k}, k \in \mathbb{N}$, is a real-valued sequence such that $0=\theta_{0}<\theta_{1}<\cdots<\theta_{k}<\cdots$ with $\theta_{k} \rightarrow \infty$ as $k \rightarrow \infty$.

Since the discrete moments of time where the argument changes its constancy may not be equally distanced, differential equations with piecewise constant argument of generalized type may lead to nonautonomous difference equations. To the best of our knowledge, 
it is the first time in the literature that a differential equation with piecewise constant argument of generalized type is reduced into a nonautonomous difference equation.

We investigate the stability of the fixed points $x=0$ and $x=\frac{a}{b}$ after reducing (5) into a nonautonomous difference equation. We discuss the existence of bounded solutions for the reduced nonautonomous difference equation. The boundedness of solutions enables us to use Lyapunov's second method developed for nonautonomous difference equations of the following type [18]:

$$
u(k+1)=f(k, u(k)), \quad k \in \mathbb{N},
$$

where $u \in \mathbb{R}^{n}, f$ is a given function of $k$ and the values $u(k)$ at $k \in \mathbb{N}$.

\section{Preliminaries}

Let us give the necessary definitions and theorems $[12,18,19]$ that will be useful in the next section. Stability definitions for difference equations are similar to the ones given for classical ordinary differential equations. Concepts of stability and their definitions for difference equations can be found in the book [18].

Let the function $f$ in (6) satisfy $f(k, 0)=0$ for all $k \in \mathbb{N}$. Then (6) admits the trivial solution $u=0$.

Definition 2.1 A function $x(t)$ is a solution of (5) on $\mathbb{R}^{+}$if

(i) $x(t)$ is continuous on $\mathbb{R}^{+}$;

(ii) the derivative $x^{\prime}(t)$ exists for $t \in \mathbb{R}^{+}$with the possible exception of the points $\theta_{k}$, $k \in \mathbb{N}$, where one-sided derivatives exist;

(iii) (5) is satisfied by $x(t)$ on each interval $\left(\theta_{k}, \theta_{k+1}\right), k \in \mathbb{N}$, and it holds for the right derivative of $x(t)$ at the points $\theta_{k}, k \in \mathbb{N}$.

Definition 2.2 A function $\phi(r)$ is said to belong to the class $\mathcal{K}$ if and only if $\phi \in$ $C\left[[0, \rho), \mathbb{R}^{+}\right], \phi(0)=0$ and $\phi(r)$ is strictly increasing in $r$.

Define $S_{\rho}=\left\{u \in \mathbb{R}^{n}:\|u\|<\rho\right\}$.

Definition 2.3 A continuous function $w: S_{\rho} \rightarrow \mathbb{R}$ is said to be positive definite if

(i) $w(0)=0$ and

(ii) $w(u)>0$ for all $u \neq 0, u \in \mathbb{S}_{\rho}$.

It is negative definite if $-w$ is a positive definite function.

Definition 2.4 A continuous function $V: \mathbb{N} \times S_{\rho} \rightarrow \mathbb{R}$ is said to be positive definite if there exists a positive definite function $w: S_{\rho} \rightarrow \mathbb{R}$ such that

(i) $V(k, 0)=0$ for all $k \in \mathbb{N}$ and

(ii) $V(k, u) \geq w(u)$ for all $k \in \mathbb{N}$ and for all $u \in S_{\rho}$.

It is negative definite if $-V(k, u)$ is a positive definite function.

Without loss of generality of our results, consider solutions of (6), whose initial value is taken at $k=0$. Let the solution $u(k)=u\left(k, u_{0}\right)$ of (6) exist and satisfy $\|u(k)\|<\rho$ for all $k \in \mathbb{N}$. Along this solution $u(k)$ of (6), the variation of the function $V(k, u)$ is defined by

$$
\begin{aligned}
\Delta V_{(6)}(k, u(k)) & =V(k+1, u(k+1))-V(k, u(k)) \\
& =V(k+1, f(k, u(k)))-V(k, u(k)) .
\end{aligned}
$$


Theorem 2.5 If there exists a positive definite scalar function $V(k, u) \in C\left[\mathbb{N} \times S_{\rho}, \mathbb{R}^{+}\right]$such that $\Delta V_{(6)}(k, u(k)) \leq 0$, then the trivial solution $u=0$ of the difference equation (6) is stable.

Theorem 2.6 If there exists a positive definite scalar function $V(k, u) \in C\left[\mathbb{N} \times S_{\rho}, \mathbb{R}^{+}\right]$ such that $\Delta V_{(6)}(k, u(k)) \leq-\alpha(\|u(k)\|)$, where $\alpha \in \mathcal{K}$, then the trivial solution $u=0$ of the difference equation (6) is asymptotically stable.

\section{Main results}

Let us reduce (5) into a difference equation. For $t \in\left[\theta_{0}, \theta_{1}\right)$, (5) takes the following form:

$$
x^{\prime}(t)=\left(a-b x\left(\theta_{0}\right)\right) x(t) .
$$

If we solve the last equation, we find $x(t)=x\left(\theta_{0}\right) \mathrm{e}^{\left(a-b x\left(\theta_{0}\right)\right)\left(t-\theta_{0}\right)}$. It follows from the continuity of the solutions that $x\left(\theta_{1}\right)=x\left(\theta_{0}\right) \mathrm{e}^{\left(a-b x\left(\theta_{0}\right)\right)\left(\theta_{1}-\theta_{0}\right)}$. For $t \in\left[\theta_{1}, \theta_{2}\right)$, we have $x\left(\theta_{2}\right)=$ $x\left(\theta_{1}\right) \mathrm{e}^{\left(a-b x\left(\theta_{1}\right)\right)\left(\theta_{2}-\theta_{1}\right)}$. Continuing the process on each interval $\left[\theta_{k}, \theta_{k+1}\right), k \geq 2$, in a similar manner, it is easy to see that

$$
x\left(\theta_{k+1}\right)=x\left(\theta_{k}\right) \mathrm{e}^{\left(a-b x\left(\theta_{k}\right)\right)\left(\theta_{k+1}-\theta_{k}\right)} .
$$

If we define a function $h: \mathbb{N} \rightarrow \mathbb{R}^{+}$given by the rule $h(k)=\theta_{k}$, then we obtain the difference equation

$$
u(k+1)=u(k) \mathrm{e}^{(a-b u(k))\left(\theta_{k+1}-\theta_{k}\right)}, \quad k \in \mathbb{N},
$$

where $u=x \circ h$. It is easy to see that the difference equation (8) has two fixed points $u=0$ and $u=\frac{a}{b}$ and the qualitative behavior of these solutions is equivalent to that of the fixed points $x=0$ and $x=\frac{a}{b}$ of (7).

First, we assume that the distance between the consecutive switching moments is any constant, i.e.,

(A1) there exists a positive constant $\theta$ such that $\theta_{k+1}-\theta_{k}=\theta$ for all $k \in \mathbb{N}$.

If the condition (A1) is fulfilled, then (8) turns into an autonomous difference equation of the following form:

$$
u(k+1)=f(u(k))=u(k) \mathrm{e}^{(a-b u(k)) \theta}, \quad k \in \mathbb{N} .
$$

Theorem 3.1 If $a<0$ then the fixed point $u=0$ of (9) is asymptotically stable and it is unstable if $a>0$. If $0<a \theta<2$ then the fixed point $u=\frac{a}{b}$ of (9) is asymptotically stable, and it is unstable if $a<0$ or $a \theta>2$.

Proof For the fixed point $u=0, f^{\prime}(0)=\mathrm{e}^{a \theta}<1$ if and only if $a<0$. For the fixed point $u=\frac{a}{b}$, the conclusion follows from the equality $f^{\prime}\left(\frac{a}{b}\right)=1-a \theta$. It is clear that $|1-a \theta|<1$ if and only if $0<a \theta<2$. Thus, the proof is completed.

Results of Theorem 3.1 can be seen in Figures 1-5.

Henceforth, we assume unless otherwise stated that the distances between the successive elements of the sequence $\theta_{k}, k \in \mathbb{N}$, are not equal. Thus, the difference $\theta_{k+1}-\theta_{k}$ is a 
Figure $1 \quad u=0$ is asymptotically stable. Solutions of (9) with $a=-0.1, b=-0.2$, and $\theta_{i}=2 i$ for different initial values $u_{0}=0.4$ (red dots), $u_{0}=0.3$ (yellow dots), $u_{0}=0.2$ (blue dots), and $u_{0}=0.1$ (green dots)

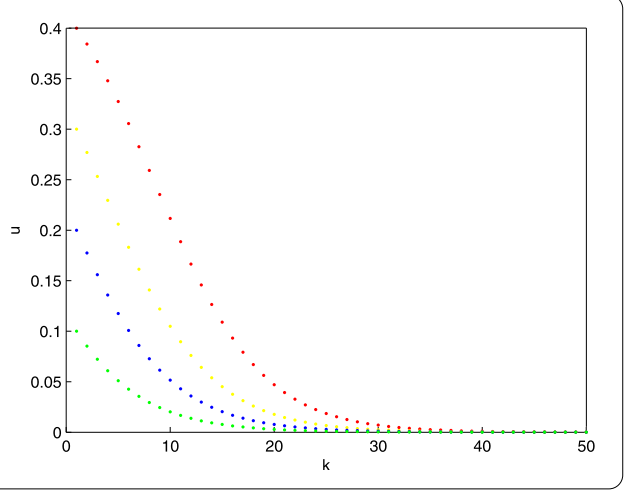

Figure $\mathbf{2} \boldsymbol{u}=\mathbf{0}$ is unstable. Solutions of (9) with $a=0.1, b=0.2$, and $\theta_{i}=2 i$ for different initial values $u_{0}=0.4$ (red dots), $u_{0}=0.3$ (yellow dots), $u_{0}=0.2$ (blue dots), and $u_{0}=0.1$ (green dots).

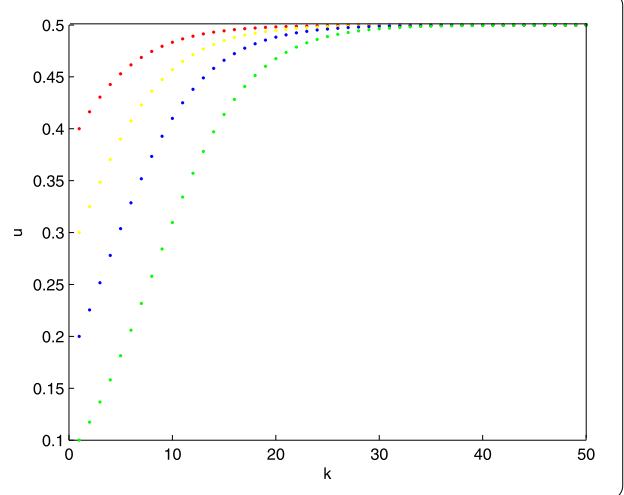

Figure $3 u=\frac{a}{b}=0.5$ is asymptotically stable. Solutions of (9) with $a=0.1, b=0.2$, and $\theta_{i}=2 i$ for different initial values $u_{0}=0.9$ (red dots), $u_{0}=0.7$ (yellow dots), $u_{0}=0.3$ (blue dots), and $u_{0}=0.1$ (green dots).

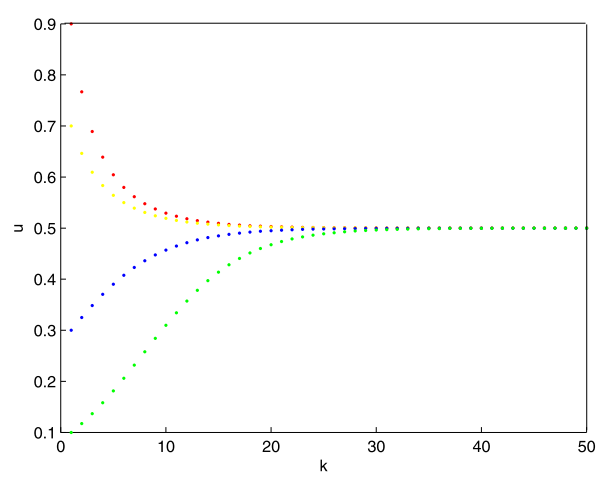

Figure $4 \boldsymbol{u}=\frac{a}{b}=\mathbf{0 . 5}$ is unstable. Solutions of (9) with $a=-0.1, b=-0.2$, and $\theta_{i}=2 i$ for different initial values $u_{0}=0.52$ (red dots), $u_{0}=0.51$ (yellow dots), $u_{0}=0.49$ (blue dots), and $u_{0}=0.48$ (green dots).

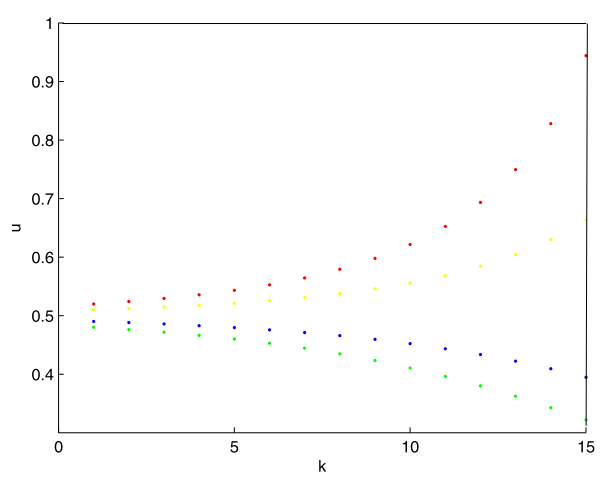


Figure $\mathbf{5} u=\frac{a}{b}=\mathbf{0 . 5}$ is unstable. Solutions of (9) with $a=1.1, b=2.2$, and $\theta_{i}=2 i$ for different initial values $u_{0}=0.52$ (red dots), $u_{0}=0.51$ (yellow dots), $u_{0}=0.49$ (blue dots), and $u_{0}=0.48$ (green dots).

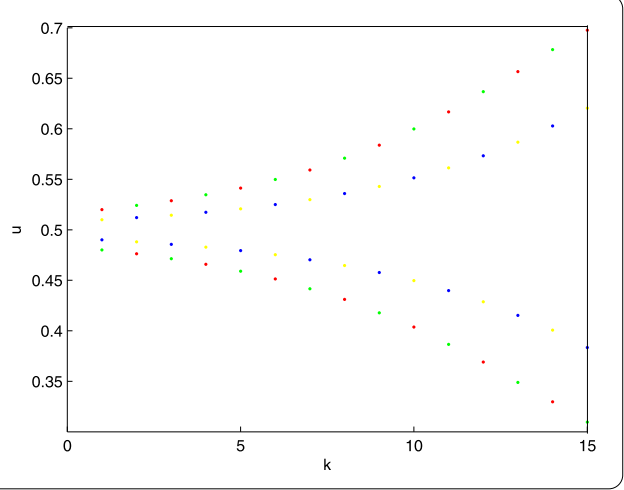

function of $k, k \in \mathbb{N}$. For convenience, we denote

$$
\theta_{k+1}-\theta_{k}=g(k), \quad k \in \mathbb{N},
$$

where $g$ is a non-constant function. Moreover, we shall use the following assumption.

(A2) There exist positive constants $\underline{\theta}$ and $\bar{\theta}$ such that $\underline{\theta} \leq \theta_{k+1}-\theta_{k}=g(k) \leq \bar{\theta}$ for all $k \in \mathbb{N}$.

Since there is no restriction on the distance between the consecutive switching moments of the argument, the reduced difference equation for (5) is of the nonautonomous type given by

$$
u(k+1)=f(k, u(k)), \quad k \in \mathbb{N},
$$

where $f(k, u(k))=u(k) \mathrm{e}^{(a-b u(k)) g(k)}$.

Let $u(k)$ be any solution of (10) with $u(0)=u_{0}>0$ for biological reasons. From now on, we shall investigate the stability of the fixed points $u=0$ and $u=\frac{a}{b}$ of the nonautonomous difference equation (10). We shall study the stability of the positive fixed point $u=\frac{a}{b}$ by means of Lyapunov's second method.

Theorem 3.2 If $a>0$ then the trivial solution $u=0$ of the difference equation (10) is unstable.

Proof Assume that $a>0$ and $u=0$ of the difference equation (10) is stable. Choose $\varepsilon=\frac{a}{2 b}$ and consider a solution $u(k), k \in \mathbb{N}$, of $(10)$ with $u(0)=u_{0}>0$. Then there exists a $\delta>0$ such that $0<u(k)<\frac{a}{2 b}$ for all $k \in \mathbb{N}$, whenever $0<u_{0}<\delta$. Provided that $0<u_{0}<\delta$, the term $a-b u(k)$ is positive for all $k \in \mathbb{N}$. Hence, it follows that

$$
u(k+1)=u(k) \mathrm{e}^{(a-b u(k))\left(\theta_{k+1}-\theta_{k}\right)} \geq u(k) \mathrm{e}^{(a-b u(k)) \theta}>u(k) .
$$

Thus, we see that the sequence $u(k), k \in \mathbb{N}$, is a strictly increasing sequence bounded above by $\frac{a}{2 b}$. Then we must have $\lim _{k \rightarrow \infty} u(k)=L \leq \frac{a}{2 b}$. Letting $k \rightarrow \infty$ in the inequality $u(k+1) \geq$ $u(k) \mathrm{e}^{(a-b u(k)) \underline{\theta}}$, we find a contradiction. This completes the proof.

Theorem 3.3 If $a<0$ then the trivial solution $u=0$ of the difference equation (10) is asymptotically stable. 
Figure $\mathbf{6} \boldsymbol{u}=\mathbf{0}$ is unstable. Solutions of (10) with $a=0.1, b=0.2$, and $\theta_{i}=\frac{i^{2}+2 i}{i+1}$ for different initial values $u_{0}=0.4$ (red dots), $u_{0}=0.3$ (yellow dots), $u_{0}=0.2$ (blue dots), and $u_{0}=0.1$ (green dots).

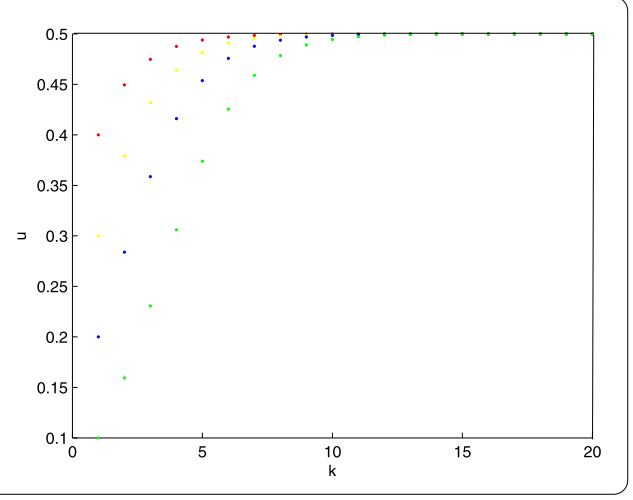

Figure $7 \boldsymbol{u}=\mathbf{0}$ is asymptotically stable. Solutions of (10) with $a=-0.5, b=-1$, and $\theta_{i}=\frac{i^{2}+2 i}{i+1}$ for different initial values $u_{0}=0.4$ (red dots), $u_{0}=0.3$ (yellow dots), $u_{0}=0.2$ (blue dots), and $u_{0}=0.1$ (green dots).

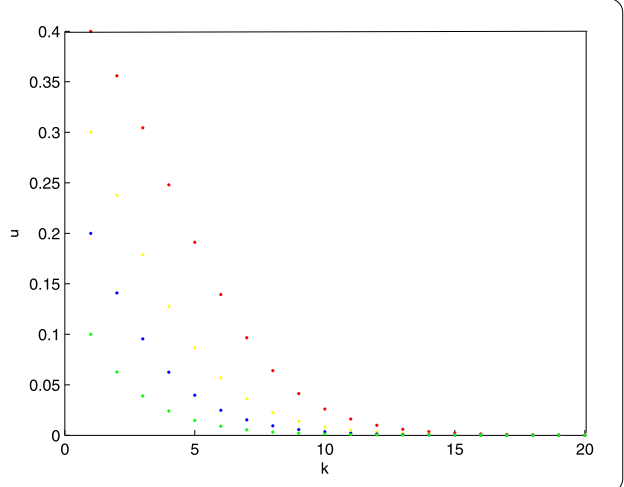

Proof Assume that $a<0$. Let $\varepsilon>0$ be given. Consider a solution $u(k), k \in \mathbb{N}$, of (10) with $u(0)=u_{0}>0$. Choose $\delta=\min \left\{\varepsilon, \frac{a}{b}\right\}$. For $0<u_{0}<\delta$, we can see that $u(k), k \in \mathbb{N}$, is a strictly decreasing sequence. Thus $u(k)<u_{0}<\delta \leq \varepsilon$ for all $k=1,2, \ldots$. This shows that $u=0$ is stable. For the same $\delta$, it can be shown easily that $\lim _{k \rightarrow \infty} u(k)=0$. Therefore, the trivial solution $u=0$ of the difference equation (10) is asymptotically stable.

Simulation results of Theorem 3.2 and Theorem 3.3 are given by Figures 6 and 7 .

Theorem 3.4 If $a<0$ then $u=\frac{a}{b}$ of the difference equation (10) is unstable.

Proof We take $a<0$ and suppose the contrary. Let $u(k), k \in \mathbb{N}$, denote the solution of (10) with $u(0)=u_{0}>0$. Then for $\varepsilon=\frac{a}{2 b}$, we can find a $\delta>0$ such that $\left|u_{0}-\frac{a}{b}\right|<\delta$ implies $\left|u(k)-\frac{a}{b}\right|<\frac{a}{2 b}$ for all $k \in \mathbb{N}$. For $\frac{a}{b}<u_{0}<\frac{a}{b}+\delta, u(k), k \in \mathbb{N}$, is a strictly increasing sequence bounded above by $\frac{3 a}{2 b}$. Thus, the solution converges to a number $L \in\left(\frac{a}{b}, \frac{3 a}{2 b}\right]$. However, when we take the limit of the inequality $u(k+1) \geq u(k) \mathrm{e}^{(a-b u(k)) \theta}$ as $k \rightarrow \infty$, it gives the contradiction $L \leq \frac{a}{b}$. For $\frac{a}{b}-\delta<u_{0}<\frac{a}{b}, u(k), k \in \mathbb{N}$, is a strictly decreasing sequence bounded below by $\frac{a}{2 b}$. Then $\frac{a}{2 b} \leq L=\lim _{k \rightarrow \infty} u(k)<\frac{a}{b}$. Letting $k \rightarrow \infty$ in the inequality $u(k+1) \leq u(k) \mathrm{e}^{(a-b u(k)) \theta}$, we find $L \geq \frac{a}{b}$, a contradiction. As a consequence, $u=\frac{a}{b}$ of the difference equation (10) is unstable.

The result of Theorem 3.4 is illustrated in Figure 8.

Now, we prove the existence of bounded solutions for (10), which will be needed in Theorem 3.9. 
Figure $8 \quad u=\frac{a}{b}=\mathbf{0 . 5}$ is unstable. Solutions of (10) with $a=-0.1, b=-0.2$, and $\theta_{i}=\frac{i^{2}+2 i}{i+1}$ for different initial values $u_{0}=0.6$ (red dots), $u_{0}=0.55$ (yellow dots), $u_{0}=0.45$ (blue dots), and $u_{0}=0.4$ (green dots).

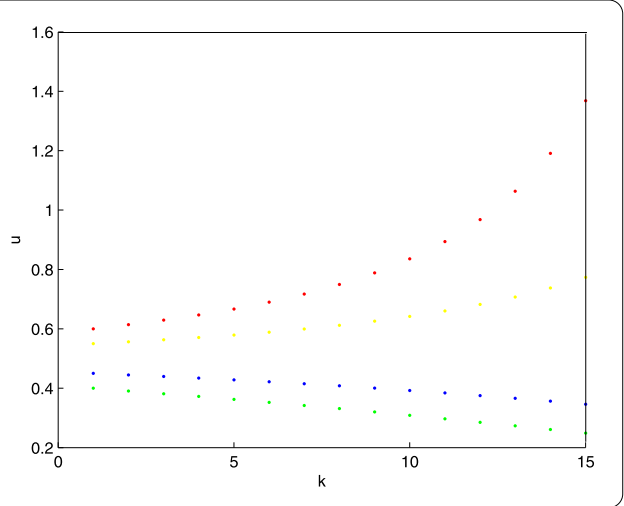

Definition 3.5 A set $\mathcal{V} \subset \mathbb{R}$ is called a positively invariant set of (10) if $u_{0} \in \mathcal{V}$ implies $u(k)=u\left(k, u_{0}\right) \in \mathcal{V}$ for all $k \in \mathbb{N}$.

Lemma 3.6 If $0<a \bar{\theta} \leq 1$, then the set $\Gamma=\left\{u \in \mathbb{R}: 0<u<\frac{a}{b}\right\}$ is positively invariant for the difference equation (10).

Proof Let $0<a \bar{\theta} \leq 1$ and $u(k), k \in \mathbb{N}$, be the solution of (10) starting at $u(0)=u_{0} \in \Gamma$. Assume that $0<u(m)<\frac{a}{b}$ for some $m \in \mathbb{N}$. We have $u(m)<u(m+1)=u(m) \mathrm{e}^{(a-b u(m))\left(\theta_{m+1}-\theta_{m}\right)} \leq$ $u(m) \mathrm{e}^{(a-b u(m)) \bar{\theta}}$. Define $H(u(m))=u(m) \mathrm{e}^{(a-b u(m)) \bar{\theta}}$. Since $0<a \bar{\theta} \leq 1$, we see that $H(u(m))<\frac{a}{b}$ on the interval $\left(0, \frac{a}{b}\right)$. Thus, we get $u(m)<u(m+1)<\frac{a}{b}$, which implies that $u(m+1) \in \Gamma$. It is seen by induction that $u(k) \in \Gamma$ for all $k \in \mathbb{N}$.

Lemma 3.7 If $0<a \bar{\theta} \leq 1$ then the set $\Gamma=\left\{u \in \mathbb{R}: 0<u \leq \kappa, \kappa \geq \frac{a}{b}\right\}$ is positively invariant for the difference equation (10).

Proof Let $0<a \bar{\theta} \leq 1$ and $u(k), k \in \mathbb{N}$, be the solution of (10) starting at $u(0)=u_{0} \in \Gamma$. If $u_{0}=$ $\frac{a}{b} \in \Gamma$ then $u(k)=\frac{a}{b} \in \Gamma$ for all $k \in \mathbb{N}$. If $u_{0} \in\left(0, \frac{a}{b}\right) \subset \Gamma$, then we know from Lemma 3.6 that $u(k) \in\left(0, \frac{a}{b}\right) \subset \Gamma$ for all $k \in \mathbb{N}$. Let us consider the case $\frac{a}{b}<u_{0} \leq \kappa$. Then either $\frac{a}{b}<$ $u(k) \leq u_{0} \leq \kappa$ for all $k \in \mathbb{N}$, or $0<u(m) \leq \frac{a}{b}$ for some natural number $m \geq 1$, which implies that $0<u(m) \leq u(k) \leq \frac{a}{b} \leq \kappa$ for all $k \geq m$. In any case, we see that the conclusion is true. Thus, the proof is completed.

Lemma 3.8 If $a \bar{\theta} \geq 1$ then the set $\Gamma=\left\{u \in \mathbb{R}: 0<u \leq \kappa, \kappa \geq \frac{1}{b \bar{\theta}} \mathrm{e}^{a \bar{\theta}-1}\right\}$ is positively invariant for the difference equation (10).

Proof Let $a \bar{\theta} \geq 1$ and $u(k), k \in \mathbb{N}$, be the solution of (10) starting at $u(0)=u_{0} \in \Gamma$. Assume that $u(m) \in \Gamma$ for some $m \in \mathbb{N}$. If $0<u(m) \leq \frac{a}{b}$, then $0<u(m) \leq u(m+1) \leq H(u(m))=$ $u(m) \mathrm{e}^{(a-b u(m)) \bar{\theta}} \leq \frac{1}{b \bar{\theta}} \mathrm{e}^{a \bar{\theta}-1} \leq \kappa$. If $\frac{a}{b} \leq u(m) \leq \kappa$, then $0<u(m+1) \leq u(m) \leq \kappa$. Thus, $u(k) \in$ $\Gamma$ for all $k \in \mathbb{N}$ by induction.

If we combine the results of Lemma 3.7 and Lemma 3.8, we can state the next result.

Corollary 1 If $a>0$ then the set $\Gamma=\left\{u \in \mathbb{R}: 0<u \leq \kappa, \kappa \geq \frac{1}{b \bar{\theta}} \mathrm{e}^{a \bar{\theta}-1}\right\}$ is positively invariant for the difference equation (10).

Theorem 3.9 If $a>0,0<u_{0} \leq \kappa$, where $\kappa>\frac{1}{b \bar{\theta}} \mathrm{e}^{a \bar{\theta}-1}$ and $b \bar{\theta}^{2}\left(\kappa-\frac{a}{b}\right)<2 \underline{\theta}\left(\ln \kappa-\ln \frac{a}{b}\right)$, then the fixed point $u=\frac{a}{b}$ of the difference equation (10) is asymptotically stable. 
Proof Let $u(k), k \in \mathbb{N}$, be the solution of (10) satisfying $0<u_{0} \leq \kappa$, where $\kappa>\frac{1}{b \bar{\theta}} \mathrm{e}^{a \bar{\theta}-1}$. Then Corollary 1 results in $0<u(k) \leq \kappa$ for all $k \in \mathbb{N}$. Define $y=u-\frac{a}{b}$. Note that $-\frac{a}{b}<y \leq \kappa-\frac{a}{b}$. It is clear that the fixed point $u=\frac{a}{b}$ of (10) is transformed to the trivial solution $y=0$ of the difference equation

$$
y(k+1)=\left(y(k)+\frac{a}{b}\right) \mathrm{e}^{-b y(k) g(k)}-\frac{a}{b} .
$$

Let $V(y)=\left[\ln \left(y+\frac{a}{b}\right)-\ln \frac{a}{b}\right]^{2}$. We see that $V$ is positive definite. For this function $V$, we have

$$
\Delta V_{(11)}(k, y(k))=-2 b g(k) y(k) \ln \frac{y(k)+\frac{a}{b}}{\frac{a}{b}}+b^{2} g^{2}(k) y^{2}(k)
$$

Since $\ln \frac{y+\frac{a}{b}}{\frac{a}{b}} \leq \frac{b}{a} y$ for $y>-\frac{a}{b}$, we have for $-\frac{a}{b}<y \leq 0$,

$$
\begin{aligned}
\Delta V_{(11)}(k, y) & \leq-2 b \underline{\theta} y \ln \frac{y+\frac{a}{b}}{\frac{a}{b}}+b^{2} \bar{\theta}^{2} y^{2} \\
& \leq-2 \frac{b^{2}}{a} \underline{\theta} y^{2}+b^{2} \bar{\theta}^{2} y^{2} \\
& =-\frac{b^{2}}{a}\left(2 \underline{\theta}-a \bar{\theta}^{2}\right) y^{2} .
\end{aligned}
$$

The assumption $b \bar{\theta}^{2}\left(\kappa-\frac{a}{b}\right)<2 \underline{\theta}\left(\ln \kappa-\ln \frac{a}{b}\right)$ implies that $a \bar{\theta}^{2}<2 \underline{\theta}$. Hence, we get $\Delta V_{(11)}(k, y) \leq-w(y)$, where $w(y)=\frac{b^{2}}{a}\left(2 \underline{\theta}-a \bar{\theta}^{2}\right) y^{2}$ is a positive definite function. Then we conclude that $\Delta V_{(11)}(k, y)$ is negative definite.

For $0 \leq y \leq \kappa-\frac{a}{b}$, consider the function $F(y)=-2 b \underline{\theta} \ln \frac{y+\frac{a}{b}}{\frac{a}{b}}+b^{2} \bar{\theta}^{2} y$. It can easily be seen that $F(y)<0, y \neq 0$, whenever $b \bar{\theta}^{2}\left(\kappa-\frac{a}{b}\right)<2 \underline{\theta}\left(\ln \kappa-\ln \frac{a}{b}\right)$ and $F(0)=0$. Since $F(y)$ is negative definite, we derive that $\Delta V_{(11)}(k, y)$ is negative definite.

Consequently, $\Delta V_{(11)}(k, y)$ is negative definite independent of the sign of $y$. Then, according to Theorem 2.6, the fixed point $y=0$ of the difference equation (11) is asymptotically stable. Thus, the fixed point $u=\frac{a}{b}$ of the difference equation (10) is asymptotically stable.

We present the numerical simulation of the result of Theorem 3.9 in Figure 9.

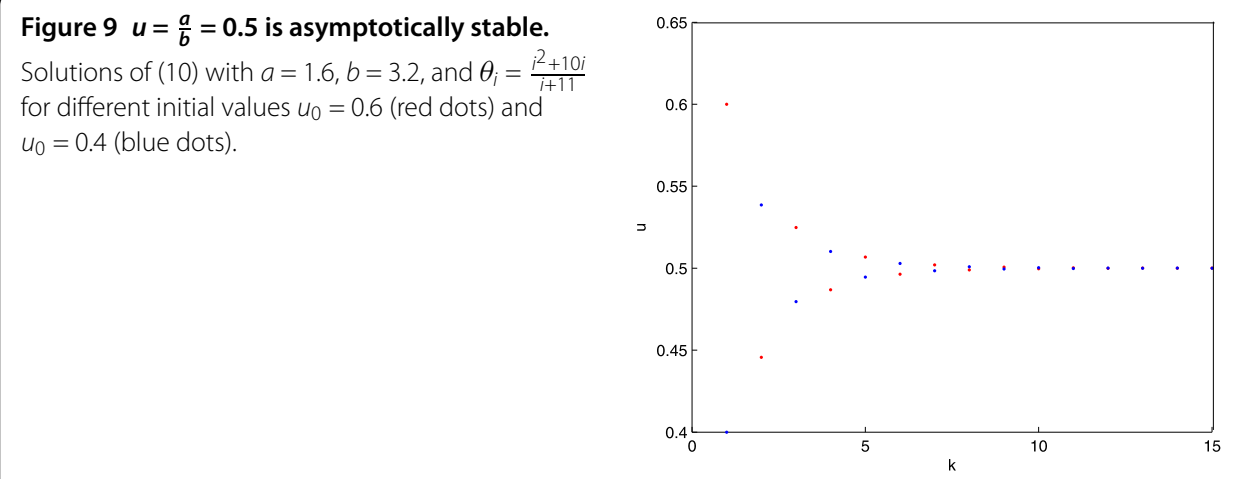




\section{Conclusion}

We see that a differential equation with piecewise constant argument, whose distance between the two consecutive switching moments is equal, can be reduced into an autonomous difference equation. However, if we have a differential equation with generalized piecewise constant argument whose switching moments are ordered arbitrarily, then it generates a nonautonomous difference equation. This fact stimulates us to study the effects of generalized piecewise constant arguments on the stability of the fixed points of the logistic equation. Our results show that the existence of a generalized piecewise constant argument influences the behavior of the solutions. As far as we know, it is the first time in the literature that one reduces a differential equation with piecewise constant argument of generalized type into a nonautonomous difference equation. This idea can be used for the investigation of differential equations with piecewise constant argument of generalized type.

\section{Competing interests}

The authors declare that they have no competing interests.

\section{Authors' contributions}

All authors contributed to the writing of the present article. They also read and approved the final manuscript.

\section{Acknowledgements}

The authors would like to express their sincere gratitude to the referees for their constructive comments and suggestions which have significantly improved the paper. This work was supported by the Scientific and Technological Research Council of Turkey (TÜBITAK), TÜBITAK 2209-A.

Received: 8 January 2015 Accepted: 27 May 2015 Published online: 10 June 2015

\section{References}

1. Ruan, S: Delay Differential Equations and Applications. Springer, Dordrecht (2006)

2. Hutchinson, GE: Circular causal systems in ecology. Ann. N.Y. Acad. Sci. 50, 221-246 (1948)

3. Petropoulou, EN: A discrete equivalent of the logistic equation. Adv. Differ. Equ. 2010, Article ID 457073 (2010)

4. Gopalsamy, K, Liu, P: Persistence and global stability in a population model. J. Math. Anal. Appl. 224, 59-80 (1998)

5. Berezansky, L, Braverman, E: Oscillation of a logistic difference equation with several delays. Adv. Differ. Equ. 2006, Article ID 82143 (2006)

6. Altıntan, D: Extension of the logistic equation with piecewise constant arguments and population dynamics. MSc thesis, Middle East Technical University (2006)

7. Muroya, Y: Persistence, contractivity and global stability in logistic equations with piecewise constant delays. J. Math. Anal. Appl. 270, 602-635 (2002)

8. Wang, Z, Wu, J: The stability in a logistic equation with piecewise constant arguments. Differ. Equ. Dyn. Syst. 14 179-193 (2006)

9. Cooke, KL, Wiener, J: Retarded differential equations with piecewise constant delays. J. Math. Anal. Appl. 99, 265-297 (1984)

10. Akhmet, MU: Integral manifolds of differential equations with piecewise constant argument of generalized type. Nonlinear Anal. 66, 367-383 (2007)

11. Akhmet, MU: On the reduction principle for differential equations with piecewise constant argument of generalized type. J. Math. Anal. Appl. 336, 646-663 (2007)

12. Akhmet, MU: Stability of differential equations with piecewise constant arguments of generalized type. Nonlinear Anal. 68, 794-803 (2008)

13. Akhmet, MU: Asymptotic behavior of solutions of differential equations with piecewise constant arguments. Appl. Math. Lett. 21, 951-956 (2008)

14. Akhmet, MU, Aruğaslan, D, Liu, X: Permanence of nonautonomous ratio-dependent predator-prey systems with piecewise constant argument of generalized type. Dyn. Contin. Discrete Impuls. Syst., Ser. A Math. Anal. 15, 37-51 (2008)

15. Aruğaslan, D, Özer, A: Stability analysis of a predator-prey model with piecewise constant argument of generalized type using Lyapunov functions. Nonlinear Oscil. 16, 452-459 (2013)

16. Akhmet, MU, Aruğaslan, D: Lyapunov-Razumikhin method for differential equations with piecewise constant argument. Discrete Contin. Dyn. Syst., Ser. A 25, 457-466 (2009)

17. Akhmet, MU, Aruğaslan, D, Yılmaz, E: Stability in cellular neural networks with a piecewise constant argument. J. Comput. Appl. Math. 233, 2365-2373 (2010)

18. Agarwal, RP: Difference Equations and Inequalities, 2nd edn. Dekker, New York (2000)

19. Miller, RK, Michel, A: Ordinary Differential Equations. Academic Press, New York (1982) 